\title{
Measuring functional dissimilarity among plots: adapting old methods to new questions
}

Sandrine Pavoine ${ }^{1, \mathrm{a}}$, Carlo Ricotta ${ }^{2, *}$

${ }^{1}$ Centre d'Ecologie et des Sciences de la Conservation (CESCO), Muséum national d'Histoire naturelle, CNRS, Sorbonne Université, 43 rue Cuvier, CP 135, 75005 Paris, France. ${ }^{2}$ Department of Environmental Biology, University of Rome 'La Sapienza', Piazzale Aldo Moro 5, 00185 Rome, Italy.

${ }^{\mathrm{a} B o t h}$ authors equally contributed to this paper.

*Correspondence author. E-mail: carlo.ricotta@uniroma1.it

Author contribution statement CR formulated the idea. SP and CR developed the methodology. SP wrote the R code and analyzed the data. SP and CR wrote the manuscript.

\section{Compliance with ethical standards}

Funding The authors received no specific funding for this work.

Conflict of interest The authors declare no conflict of interest.

Abstract Ecologists routinely use dissimilarity measures between pairs of plots to explore the complex mechanisms that drive community assembly. Traditional dissimilarity measures usually quantify plot-to-plot dissimilarity based either on species presences and absences within plots or on species abundances, thus assuming that all species are equally and maximally distinct from one another. However, the value of dissimilarity measures that incorporate information on functional differences among species is becoming increasingly recognized. Since these 'functional dissimilarity measures' have been developed for capturing new aspects of plot-to-plot dissimilarity, they have usually little to do with more traditional measures based solely on species incidence or abundance data. In this paper we introduce a general method for adapting a large family of traditional dissimilarity coefficients to the measurement of functional differences among plots. The behavior of the proposed method, for which we provide a simple $\mathrm{R}$ function, was evaluated with published data on plant communities in a coastal marsh plain in Algeria. As shown by the worked example, our proposal produces a coherent framework for summarizing functional dissimilarity among plots. Being based on a generalization of classical dissimilarity measures with well-known properties, this new family of functional dissimilarity indices also has a great potential for future theoretical and applied developments in this field of research.

Keywords: Branching property; Bray-Curtis dissimilarity; Species ordinariness; Sum property. 


\section{Introduction}

Ecologists have developed a multitude of (dis)similarity measures between pairs of plots (or communities, assemblages, relevés, sites, quadrats, etc.) for exploring various aspects of the complex mechanisms that drive community assembly (see e.g. Orlóci 1978; Podani 2000; Legendre and Legendre 2012). These measures usually quantify plot-to-plot dissimilarity based either on species incidence or abundance within plots, thus assuming that all species are equally and maximally distinct from one another, while neglecting information on functional differences among species.

More recently, a number of 'functional dissimilarity measures' have been proposed for summarizing different facets of functional differences among plots (Rao 1982; Clarke and Warwick 1998; Izsák and Price 2001; Champely and Chessel 2002; Pavoine et al. 2004; Chiu et al. 2014; Pavoine and Ricotta 2014; Ricotta et al. 2016). Such new dissimilarity measures incorporate information on the species functional traits. Therefore, they are expected to correlate more strongly with ecosystem-level processes, as species influence these processes via their traits (Mason and de Bello 2013). Since functional dissimilarity measures have been developed for capturing new aspects of ecological differences among plots, they are usually not directly related to more traditional measures of species turnover which are based solely on species incidence or abundance data.

The aim of this paper is thus to introduce a methodological framework for adapting a large family of traditional dissimilarity coefficients to the measurement of functional differences among plots. The main advantage of this approach is that being based on a generalization of well-known dissimilarity measures which have been extensively used in ecology for a long time, these new functional measures benefit from decades of research on multivariate resemblance.

\section{Methods}

\subsection{A general family of traditional dissimilarity measures}

Let $U$ and $V$ be two plots where $x_{U j}$ and $x_{V j}$ are the abundance values of species $j$ in plots $U$ and $V$, respectively, and $N$ is the total number of species sampled in both plots (i.e. the species for which $\left.\min \left\{x_{U j}, x_{V j}\right\}>0\right)$. A desirable property for a dissimilarity coefficient is the so-called 'sum property' (Ricotta and Podani 2017). That is, its ability to be additively partitioned into specieslevel contributions thus enabling to emphasize the relevance of single species to plot-to-plot dissimilarity.

Among the many dissimilarity measures that conform to the sum property, the Canberra distance (Lance and Williams 1967) is obtained by standardizing separately for each species the absolute difference in species abundances by the sum of the abundances in both plots: 
$79 \quad C D=\sum_{j=1}^{N} \frac{\left|x_{U j}-x_{V j}\right|}{\left(x_{U j}+x_{V j}\right)}$

80

The Canberra distance is bounded between 0 and $N$. Therefore, a normalized measure in the

83

$84 \quad N C=\frac{1}{N} \sum_{j=1}^{N} \frac{\left|x_{U j}-x_{V j}\right|}{\left(x_{U j}+x_{V j}\right)}$

85

86

On the other hand, the dissimilarity coefficient proposed by Bray and Curtis (1957), one of the most popular measures of compositional dissimilarity among ecologists, implies normalization of the sum of species-wise differences by the total abundance of species in both plots:

89

90

$$
B C=\frac{\sum_{j=1}^{N}\left|x_{U j}-x_{V j}\right|}{\sum_{j=1}^{N}\left(x_{U j}+x_{V j}\right)}
$$

91

92

93

94

$95 \quad B C=\sum_{j=1}^{N} \frac{\left|x_{U j}-x_{V j}\right|}{\sum_{k=1}^{N}\left(x_{U k}+x_{V k}\right)}=\sum_{j=1}^{N} w_{j} \frac{\left|x_{U j}-x_{V j}\right|}{\left(x_{U j}+x_{V j}\right)}$

96

97

98

$99 \quad w_{j}=\left(x_{U j}+x_{V j}\right) / \sum_{k=1}^{N}\left(x_{U k}+x_{V k}\right)$

100

101 with $0 \leq w_{j} \leq 1$ and $\sum_{j=1}^{N} w_{j}=1$.

103 we can rewrite $\mathrm{BC}$ as:

where

\section{8}

00

102 As shown by Eq. (4), the Bray-Curtis dissimilarity can be expressed as a normalized form of the $104\left|x_{U j}-x_{V j}\right| /\left(x_{U j}+x_{V j}\right)$ is weighted by the relative abundance of $j$ in plots $U$ and $V$. Based on the

To emphasize the relationship between the Bray-Curtis dissimilarity and the Canberra distance, 03 Canberra distance in which the contribution of species $j$ to overall dissimilarity 
$D_{M S}=\sum_{j=1}^{N} \pi_{j} \frac{\left|x_{U j}-x_{V j}\right|}{\max \left\{x_{U j}, x_{V j}\right\}}$

$$
D_{E V E}=\sum_{j=1}^{N} \pi_{j}\left(1-E V E_{j}\right)
$$
obtained with Pielou's evenness: 
where $H_{j}$ is the Shannon entropy of species $j: H_{j}=-\frac{x_{U j}}{x_{U j}+x_{V j}} \log \left(\frac{x_{U j}}{x_{U j}+x_{V j}}\right)-\frac{x_{V j}}{x_{U j}+x_{V j}} \log \left(\frac{x_{V j}}{x_{U j}+x_{V j}}\right)$.

In the next sections, we will show how to apply this family of dissimilarity coefficients to the measurement of functional dissimilarity among plots.

\subsection{A new family of functional dissimilarity measures}

Regardless of how pairwise functional differences among species $i$ and $j$ are calculated, they are usually represented by symmetric dissimilarity coefficients $\delta_{i j}$ with $\delta_{i j}=\delta_{j i}$ and $\delta_{i i}=0$. If $\delta_{i j}$ is bounded in the range $[0,1]$, we can derive a corresponding similarity coefficient $\sigma_{i j}=1-\delta_{i j}$ as the complement of $\delta_{i j}$. Note that dissimilarity measures with an upper bound $\delta_{\max }>1$ can be normalized by dividing each term by $\delta_{\max }$, while for dissimilarity measures without an upper bound we can still get a locally normalized dissimilarity in the range $[0,1]$ by dividing each term by the maximum value in the data set.

Combining species abundances $x_{U j}$ and between-species similarities $\sigma_{i j}$, Leinster and Cobbold (2012) defined the 'ordinariness' of species $j$ as the abundance of all species in plot $U$ that are functionally similar to $j$ such that:

$$
z_{U j}=\sum_{i=1}^{N} x_{U i} \sigma_{i j}
$$

where $z_{U j}$ is the abundance of all species that are functionally similar to $j$ (including $j$ itself). For species $j, z_{U j}$ thus measures the commonness of all individuals in plot $U$ that support the functions associated with $j . z_{U j}$ ranges from $x_{U j}$ if all species $i \neq j$ are maximally dissimilar from $j$, such that $\sigma_{i j}=0$, to $\sum_{j=1}^{N} x_{U j}$ (i.e. the total species abundance in plot $U$ ) if all species $i \neq j$ are functionally identical to $j$ such that $\sigma_{i j}=1$. Hence, the abundance of species similar to $j$ is at least as great as the abundance of $j$ itself.

Given two functionally identical plots $U$ and $V$, we have that for each species in $U$ and $V$, the ordinariness $z_{U j}=\sum_{i=1}^{N} x_{U i} \sigma_{i j}$ in plot $U$ is equal to the corresponding value $z_{V j}=\sum_{i=1}^{N} x_{V i} \sigma_{i j}$ in plot $V$. In other words, for two functionally identical plots, the abundance of the species similar to $j$ in 
plot $U$ is equal to the abundance of the species similar to $j$ in plot $V$ (for definitions and proofs see Appendix 1). In contrast, for two maximally distinct assemblages, either $z_{U j}$ or $z_{V j}$ is equal to zero, meaning that the species in plot $U$ do not have any functional analogue in plot $V$.

Hence, in principle, we could calculate a measure of functional dissimilarity among plots with any of the many traditional dissimilarity measures developed for species abundance data by simply replacing the species abundances $x_{U j}$ and $x_{V j}$ in both plots with their corresponding species ordinariness $z_{U j}$ and $z_{V j}$. The resulting measures meet the foremost requirements for a dissimilarity coefficient in the range [0, 1]: for two maximally dissimilar plots (i.e. two plots with no species in common and $\sigma_{i j}=0$ for species $i$ belonging to the first plot and species $j$ to the second plot), the measure takes the value one, whereas for two functionally identical plots the measure takes the value zero (see Pavoine and Ricotta 2014).

\subsection{An additional requirement for functional dissimilarity}

A potential drawback of this approach is that the dissimilarity indices obtained by substituting the species abundances $x_{U j}$ and $x_{V j}$ in $U$ and $V$ with the species ordinariness $z_{U j}$ and $z_{V j}$ tend to overemphasize the relevance of the most functionally redundant species. For example, consider two plots $U$ and $V$ and two maximally dissimilar species $i$ and $j$ with $\sigma_{i j}=0$. Plot $U$ contains species $i$ with abundance $x_{U i}=5$ and species $j$ also with abundance $x_{U j}=5$, while plot $V$ contains only species $j$ with abundance $x_{V j}=10$ :

\begin{tabular}{|l|c|c|}
\hline & $i$ & $j$ \\
\hline Plot $U$ & 5 & 5 \\
\hline Plot V & 0 & 10 \\
\hline
\end{tabular}

Since the two species are maximally dissimilar, their ordinariness is identical to their abundances: $x_{U i}=z_{U i}=5, x_{U j}=z_{U j}=5$, and $x_{V j}=z_{V j}=10$. Therefore, using the species ordinariness for calculating the functional dissimilarity between $U$ and $V$ with the Bray-Curtis coefficient (see Eq. 3), we obtain $B C_{z}=0.5$.

However, if we split species $i$ into two functionally identical species $i^{\prime}$ and $i^{\prime \prime}$, each with abundance $x_{U i^{\prime}}=x_{U i^{\prime \prime}}=2.5$ and $\sigma_{i^{\prime} i^{\prime \prime}}=1$ : 


\begin{tabular}{|l|c|c|c|}
\hline & $i^{\prime}$ & $i^{\prime \prime}$ & $j$ \\
\hline Plot $U$ & 2.5 & 2.5 & 5 \\
\hline Plot $V$ & 0 & 0 & 10 \\
\hline
\end{tabular}

191

we have $z_{U i^{\prime}}=z_{U i^{\prime \prime}}=5, z_{U j}=5$ and $z_{V j}=10$. Accordingly, $B C_{z}=0.6$, whereas, intuitively, the functional dissimilarity between $U$ and $V$ should not change if one species is replaced by two identical species with the same total abundance.

More generally, irrespective of the values of the pairwise similarities among species pairs, the species ordinariness overemphasizes the role of the functionally redundant species in both plots. If the species in $U$ are functionally similar to the species in $V$, the similarity between $U$ and $V$ will be exaggerated. To the contrary, if the species in $U$ are functionally different from the species in $V$, the similarity between $U$ and $V$ will be excessively reduced.

Therefore, a meaningful measure of functional dissimilarity between pairs of plots needs to conform to the following branching requirement: the value of functional dissimilarity among a pair of plots $U$ and $V$ should not change if a given species $j$ is replaced by two functionally identical species with the same total abundance of $j$ in $U$ and $V$.

In Appendix 1, we show that a special case of the general family of dissimilarity coefficients $D_{\pi}$ conforms to this additional requirement. If the single-species dissimilarities $d$ in Eq. (7) are calculated from the species ordinariness $z_{U j}$, while the weighting factors $w_{j}$ are calculated from the original species abundances $w_{j}=\left(x_{U j}+x_{V j}\right) / \sum_{k=1}^{N}\left(x_{U k}+x_{V k}\right)$, we get a family of measures:

$$
D_{w}=\sum_{j=1}^{N} w_{j} d\left(z_{U j}, z_{V j}\right)
$$

which conforms to the branching requirement for functional dissimilarity coefficients (proof in Appendix 1):

Take for example the generalized version of the Bray-Curtis coefficient. If this measure is calculated as $B C_{w}=\sum_{j=1}^{N} w_{j}\left|z_{U j}-z_{V j}\right| /\left(z_{U j}+z_{V j}\right)$, it is easily shown that $B C_{w}$ conforms to the branching requirement. The same holds for all functional dissimilarity coefficients that can be expressed according to Eq. (12).

\section{Worked example}

We analyzed the functional composition of plant communities in the coastal marsh plain of La Mafragh ( $\left.36^{\circ} 48^{\prime} \mathrm{N}-008^{\circ} 00^{\prime} \mathrm{E}\right)$ in Algeria. Detailed information on the data set can be found in de 
Bélair (1981) and Pavoine et al. (2011). The area is a basin filled by alluvial and colluvial deposits and furrowed by rivers. The lowest parts are composed of large and small marshes. The study area is bounded by dunes with a narrow connection (Oued Mafragh) to the Mediterranean Sea in the north (north is at the top of the panels), by Numidian clay-sandstone mountains in the south, by a river (Oued El Kebir) in the east, and by an irrigated agricultural zone in the west. It is about $15 \mathrm{~km}$ large from east to west and $8 \mathrm{~km}$ from north to south.

97 study sites were regularly distributed on the plain. de Bélair (1981) collected species abundances and environmental data (de Bélair 1981), and Pavoine et al. (2011) collected species traits from the literature. As in Pavoine et al. (2011), we focused our analyses on four traits: life cycle (categorical multichoice variable with four levels [perennial; annual; biennial; seasonal]), pollination (categorical multichoice variable with three levels [autogamous, entomogamous, anemogamous]), presence of spiky structures (ordinal variable with three levels $[0=$ no; $1=$ sometimes; $2=$ yes $]$ ), and hairy leaves (ordinal variables with three levels $[0=$ no; $1=$ sometimes; 2 $=$ yes]). We calculated the functional dissimilarities between species as the average dissimilarity over all traits (see Pavoine et al. 2009). We used the Sørensen index for categorical multichoice traits and the Euclidean distance on rank-transformed ordinal variables.

To obtain species-based dissimilarities among plots, we applied the traditional Bray-Curtis dissimilarity (Eq. 4) to the species relative abundances. Then, to obtain functional dissimilarities among plots, we applied the functional version of the Bray-Curtis dissimilarity to the species ordinariness $z_{U j}$ (see Eq. 12). We then analyzed these plot-to-plot dissimilarities with Principal Coordinate Analysis (PCoA) after Cailliez (1983) transformation. This operation consists in adding the smallest constant to all dissimilarities to make the resulting dissimilarity matrix Euclidean. We finally compared the results obtained from the PCoA with the spatial distribution of eight environmental variables (Fig. 1): elevation [m], clay [\%], silt [\%], sand [\%], $\mathrm{K}_{2} \mathrm{O}[\% \mathrm{o}], \mathrm{Mg}^{2+}[\mathrm{mEq} /$ $100 \mathrm{~g}], \mathrm{Na}^{+}[\mathrm{mEq} / 100 \mathrm{~g}], \mathrm{K}^{+}[\mathrm{mEq} / 100 \mathrm{~g}]$. The $\mathrm{R}$ scripts developed for this analysis are provided in Appendix 2.

\section{Results}

We retained the first axis of the PCoA applied to plot-to-plot functional dissimilarities (Fig. 2) as its eigenvalue is about five-fold that of the second axis (Fig. 3). The high number of eigenvalues close to 0.006 in this analysis (Fig. 3A) is due to the Cailliez transformation used to render the dissimilarities Euclidean. Similar patterns were also obtained using non-metric Multidimensional Scaling (Appendix 2), meaning that the results were not affected by the ordination method used for analyzing the data. According to Figure 1 and 2, the negative part of the PCoA axis corresponded 
mainly to plots at lower elevation in the middle of the study area where clay-rich soils had high concentrations of $\mathrm{K}+, \mathrm{Mg}++, \mathrm{Na}+$ and $\mathrm{K}_{2} \mathrm{O}$, indicating high salinity. On the other hand, plots with positive coordinates tended to be located at higher elevations on soils with comparatively higher proportions of silt and sand and with lower salinity levels, mostly on the West side of the plain.

Looking only at species-based dissimilarities, without considering trait data, the first eigenvalue of the PCoA is about twice as large as the second eigenvalue (Fig. 3B). In that case, the sites were roughly divided into two groups: those in the central part of the plain and those on the East and West side (Fig. 4A). Compared to the smoother gradient obtained with trait data, this pattern is more sensitive to changes in the elevation of the study area and less to the salinity gradient.

We finally compared the plot-to-plot dissimilarities obtained solely from species abundances with the corresponding functional dissimilarities. As expected, relaxing the constraint that all species are equally and maximally distinct from one another, trait-based dissimilarities were always lower than species-based dissimilarities (Fig. 4B). The functional dissimilarities among sites with no species in common (species-based dissimilarity $=1$ ) varied between about 0 and 0.5 (Fig. 4B).

\section{Discussion}

Ecological data are often multivariate of high dimensionality. Therefore, no single measure adequately summarizes all aspects of (functional) dissimilarity among plots. In order to assess differences in the functional organization of species assemblages, a multifaceted approach is needed. In this paper, we thus proposed a new method for adapting traditional dissimilarity coefficients to the measurement of functional differences among plots. The resulting measures generalize species dissimilarity to include functional information. As such, they allow plot-to-plot comparisons at different levels of ecological complexity (i.e. compositional vs. functional dissimilarity), while avoiding confounding effects which may be due to differences in the calculation of the indices (Ricotta and Pavoine 2015).

The basic unit for the calculation of functional dissimilarity is the species ordinariness $z_{U j}$. In Appendix 3 we show that, in the special case where the species ordinariness are calculated from the relative abundances $p_{U j}=x_{U j} / \sum_{j=1}^{N} x_{U j}$ of each species, the resulting values of $z_{U j}$ are tightly related to the notion of species rarity used by Patil and Taillie (1982) for providing a generalized definition of diversity measures. As such, our proposal may provide a starting point for developing a unified framework for the summarization of functional diversity and dissimilarity.

Note that species ordinariness was already used by Ricotta et al. (2015) for developing a single Bray and Curtis-like index of functional dissimilarity and by Ricotta and Pavoine (2015) for 
constructing a family of functional dissimilarity measures based on a generalization of the classical $2 \times 2$ contingency table to include species abundances and interspecies resemblances. However, these measures do not conform to the newly proposed branching requirement for functional dissimilarity coefficients.

Given two assemblages $U$ and $V$, the major difference between classical species abundance values $x_{U j}$ and the corresponding species ordinariness $z_{U j}$ is that the former are stand-alone quantities, which are independent of the abundances of the other species in $U$ and $V$, whereas the ordinariness of species $j$ in plot $U$ depends not only on its abundance $x_{U j}$, but also on its functional resemblance with the other species in $U$ and $V$. Hence, using species ordinariness for the calculation of plot-to-plot functional dissimilarity, we implicitly assume that species with similar traits are likely to support similar functions, such that the selection of the most appropriate traits needs to be explicitly targeted on the ecological process of interest. Also, although the formulation of species uniqueness does not consider within-species differentiation, it is commonly agreed that species differences in trait values are related to different mechanisms of resource use, such that the higher the species overlap in functional space, the higher the species are expected to support similar functions. Therefore, using dissimilarity measures based on functional overlap among species (Lepš et al. 2006; Blonder et al. 2014), will allow us to include within-species differentiation in the calculation of plot-to-plot functional dissimilarity.

To conclude, we hope our proposal will prove fruitful for summarizing plot-to-plot functional dissimilarity in a meaningful way and for getting insights into the complex ecological mechanisms that drive community structure.

\section{Supporting Information}

Appendix 1. Definitions and Proofs

Appendix 2. R scripts

Appendix 3. On the relationship between species ordinariness and species rarity 


\section{References}

Blonder, B, Lamanna, C, Violle, C, Enquist, BJ (2014) The n-dimensional hypervolume. Global Ecol Biogeogr 23: 595-609

Bray, JR, Curtis, JT (1957) An ordination of the upland forest communities of southern Wisconsin. Ecol Monogr 27: 325-349

Cailliez, F (1983) The analytical solution to the additive constant problem. Psychometrika 48: 305308

Champely, S, Chessel, D (2002) Measuring biological diversity using Euclidean metrics. Environ Ecol Stat 9: 167-177

Chiu, C-H, Jost, L, Chao, A (2014) Phylogenetic beta diversity, similarity, and differentiation measures based on Hill numbers. Ecol Monogr 84: 21-44

Clarke, KR, Warwick, RM (1998) A taxonomic distinctness index and its statistical properties. J Appl Ecol 35: 523-531

de Bélair, G (1981) Biogéographie et aménagement: la plaine de La Mafragh (Annaba, Algérie). PhD Thesis, Université Paul Valéry, Montpellier

Izsák, C, Price, RG (2001) Measuring $\beta$-diversity using a taxonomic similarity index, and its relation to spatial scale. Mar Ecol Prog Ser 215: 69-77

Lance, GN, Williams, WT (1967) Mixed data classificatory programs. I. Agglomerative systems. Aust Comput J 1: 15-20

Legendre, P, Legendre, L (2012) Numerical Ecology. Elsevier, Amsterdam

Leinster, T, Cobbold, CA (2012) Measuring diversity: the importance of species similarity. Ecology 93: 477-89

Lepš, J, de Bello, F, Lavorel, S, Berman, S (2006) Quantifying and interpreting functional diversity of natural communities: practical considerations matter. Preslia 78: 481-501

Mason, NWH, de Bello, F (2013) Functional diversity: a tool for answering challenging ecological questions. J Veg Sci 24: 777-780

Orlóci, L (1978) Multivariate Analysis in Vegetation Research. Junk, The Hague, NL

Patil, GP, Taillie, C (1982) Diversity as a concept and its measurement. J Am Stat Ass 77: 548-561

Pavoine, S, Dufour, A-B, Chessel, D (2004) From dissimilarities among species to dissimilarities among communities: a double principal coordinate analysis. J Theor Biol 228: 523-537

Pavoine, S, Ricotta, C (2014) Functional and phylogenetic similarity among communities. Methods Ecol Evol 5: 666-675 
346 Pavoine, S, Vallet, J, Dufour, A-B, Gachet, S, Daniel, H (2009) On the challenge of treating various 347 types of variables: application for improving the measurement of functional diversity. Oikos 118: $348 \quad 391-402$

349 Pavoine, S, Véla, E, Gachet, S, de Bélair, G, Bonsall, MB (2011) Linking patterns in phylogeny, 350 traits, abiotic variables and space: a novel approach to linking environmental filtering and plant 351 community assembly. J Ecol 99: 165-175

352 Podani, J (2000) Introduction to the Exploration of Multivariate Biological Data. Backhuys, Leiden, $353 \quad$ NL

354 Rao, CR (1982) Diversity and dissimilarity coefficients: a unified approach. Theor Pop Biol 21: 24$355 \quad 43$

356 Ricotta, C (2018) A family of (dis)similarity measures based on evenness and its relationship with 357 beta diversity. Ecol Complex 34: 69-73

358 Ricotta, C, Bacaro, G, Pavoine, S (2015) A cautionary note on some phylogenetic dissimilarity 359 measures. J Plant Ecol 8: 12-16

360 Ricotta, C, Pavoine, S (2015) Measuring similarity among plots including similarity among species: 361 an extension of traditional approaches. J Veg Sci 26: 1061-1067

362 Ricotta, C, Podani, J (2017) On some properties of the Bray-Curtis dissimilarity and their ecological 363 meaning. Ecol Complex 31: 201-205

364 Ricotta, C, Podani, J, Pavoine, S (2016) A family of functional dissimilarity measures for presence 365 and absence data. Ecol Evol 6: 5383-5389 
(A) Clay (\%)

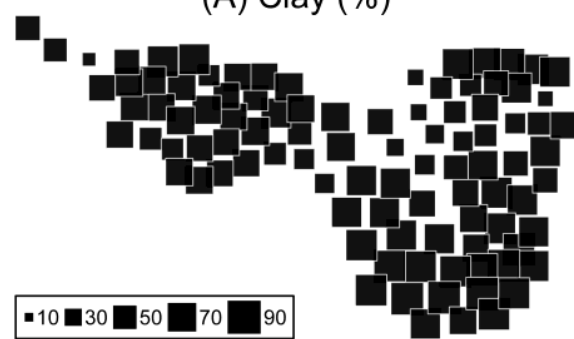

(C) Sand (\%)
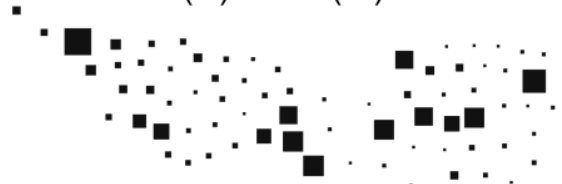

\begin{tabular}{|l|l|l|l|l|}
\hline$-10 \square 30$ & 50 & $\square 0$ & & 90 \\
\hline
\end{tabular}

(E) $\mathrm{Mg}^{++}(\mathrm{mEq} / \mathrm{100g})$

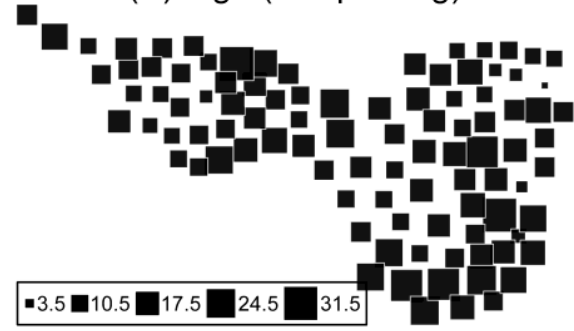

(G) $\mathrm{K}_{2} \mathrm{O}(\% \mathrm{o})$

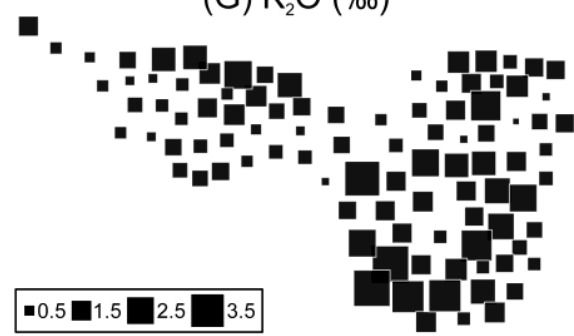

(B) Silt (\%)

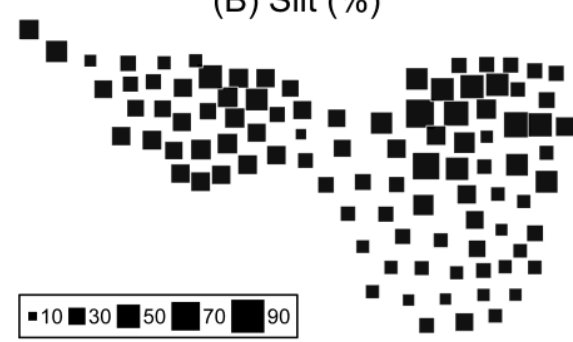

(D) $\mathrm{K}^{+}(\mathrm{mEq} / 100 \mathrm{~g})$

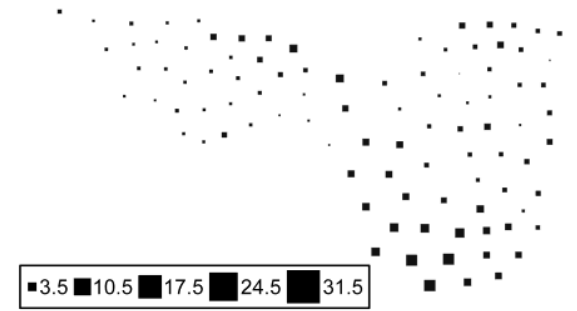

(F) $\mathrm{Na}^{+}(\mathrm{mEq} / 100 \mathrm{~g})$

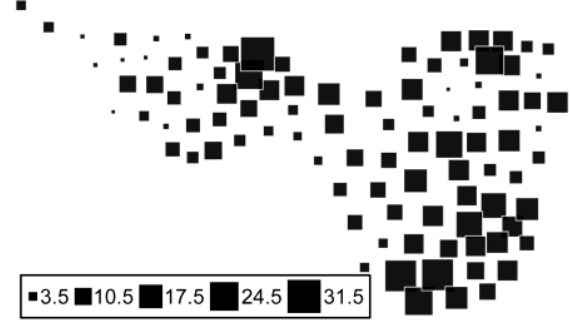

(H) Elevation (m)

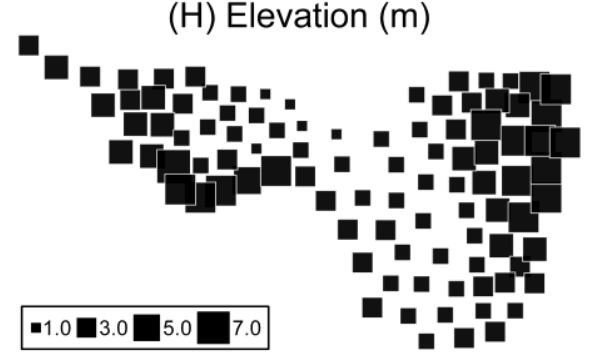

Figure 1. Environmental patterns in the coastal marsh plain of La Mafragh. The locations of the squares correspond to the geographic positions of the sample plots. The square size provides the level of the environmental variables. 
(A)

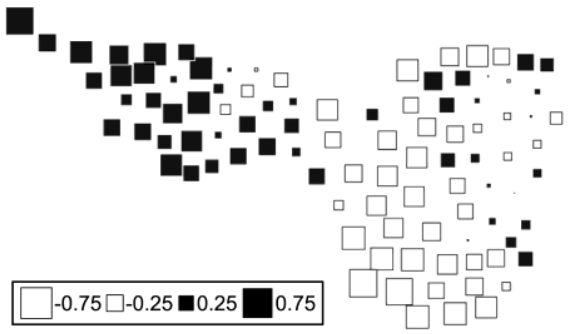

(B)

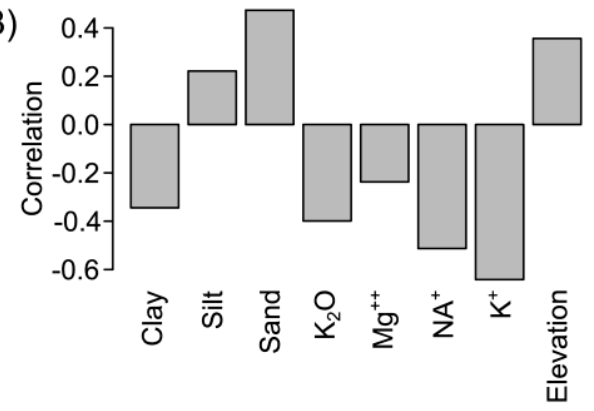

Figure 2. Results of the principal coordinate analysis applied to plot-to-plot functional dissimilarities after Cailliez transformation. (A) first axis of the PCoA applied to plot-to-plot functional dissimilarities. (B) Bar plot of the Spearman correlations between the first axis of the PcoA and the environmental variables.

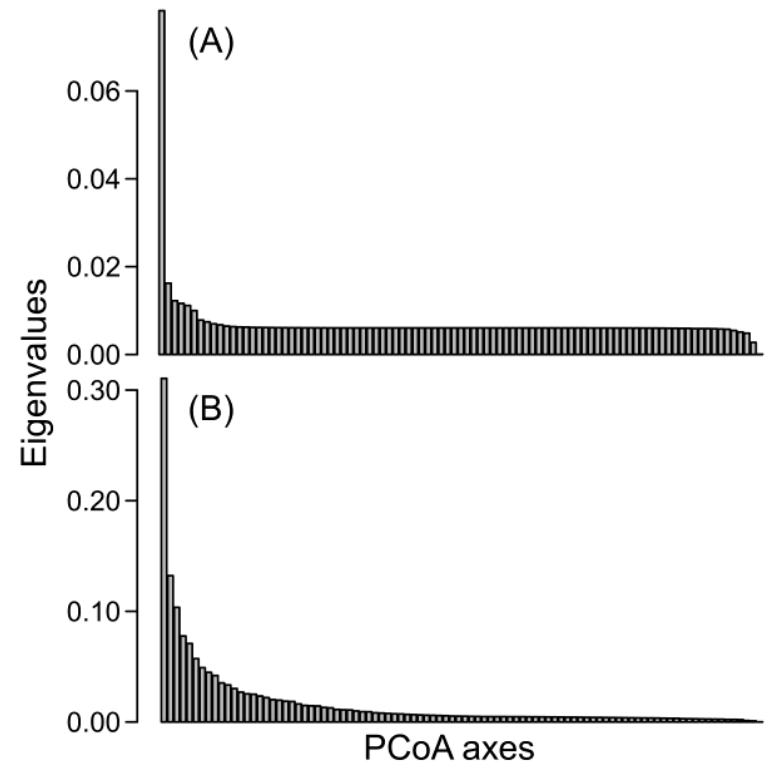

Figure 3. Eigenvalue bar plot of the principal coordinate analysis applied to (A) plot-to-plot functional dissimilarities and (B) plot-to-plot species-based dissimilarities, after Cailliez transformation. 

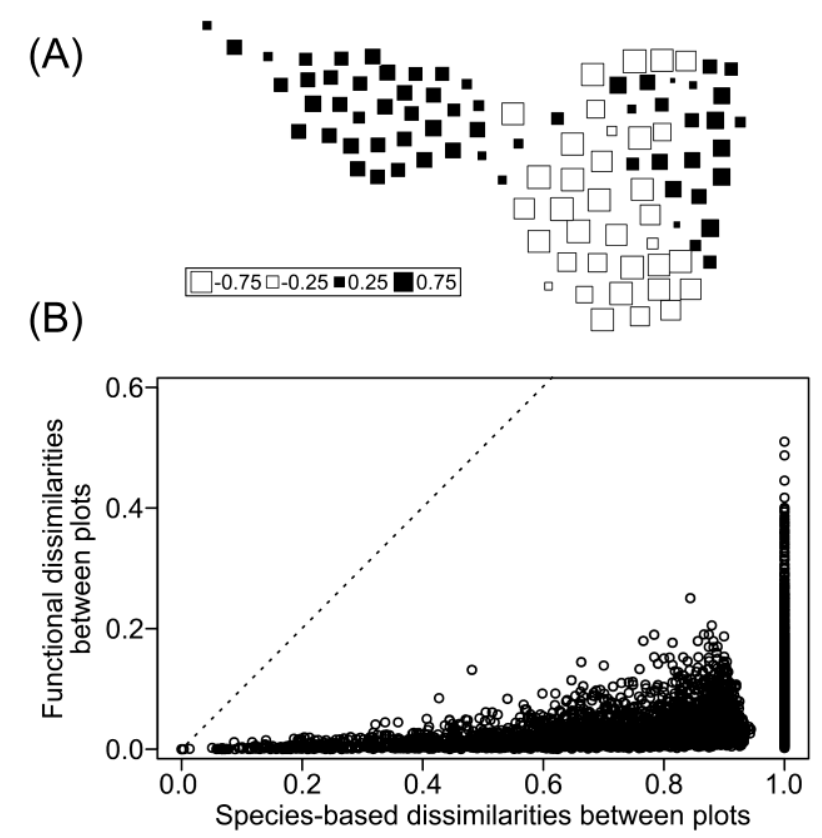

Figure 4. Species-based dissimilarities among plant communities in the coastal marsh plain of La Mafragh. (A) First axis of the principal coordinate analysis applied to species-based dissimilarities among plots. (B) Relationship between functional and species-based dissimilarities among plots. The dashed line corresponds to the theoretical scenario where species-based and functional dissimilarities would have equal values.

\section{Appendix 1. Definitions and Proofs}

Hereafter, we consider two plots $U$ and $V$, where $x_{U j}$ and $x_{V j}$ are the abundance values of species $j$ in plots $U$ and $V$, respectively, $N$ is the number of species of the pooled pair of plots (i.e. all species with non-zero abundance in at least one plot), and $\sigma_{i j}$ is a symmetric similarity coefficient between species $i$ and $j$ bounded in the range $[0,1]$ with $\sigma_{i j}=\sigma_{j i}$ and $\sigma_{i i}=0$.

Two species $i$ and $j$ will be said functionally identical if their functional similarity is maximal: $\sigma_{i j}=1$.

Two plots $U$ and $V$ with no shared species are said functionally identical if for any species $j$ (or any set of functionally identical species $j, j^{\prime}, j^{\prime \prime}, \ldots$ ) in plot $U$, a species $k$ (or a set of functionally identical species $k, k^{\prime}, k^{\prime \prime}, \ldots$ ) exist in plot $V$ such that $x_{U j}=x_{V k}$ and $\sigma_{j k}=1$ (or $x_{U j}+x_{U j^{\prime}}+x_{U j^{\prime \prime}}+\ldots=x_{V k}+x_{V k^{\prime}}+x_{V k^{\prime \prime}}+\ldots$ and $\sigma_{j k}=1$, in case of functionally identical species in $U$ and/or in $V$ ), and if similarly for any species $k$ (or a set of functionally identical species $k, k^{\prime}, k^{\prime \prime}$ ,$\ldots$ ) in plot $V$, a species $j$ (or any set of functionally identical species $j, j^{\prime}, j^{\prime \prime}, \ldots$ ) exist in plot $U$ such that $x_{U j}=x_{V k}$ and $\sigma_{j k}=1$ (or $x_{U j}+x_{U j^{\prime}}+x_{U j^{\prime \prime}}+\ldots=x_{V k}+x_{V k^{\prime}}+x_{V k^{\prime \prime}}+\ldots$ and $\sigma_{j k}=1$, in case of functionally identical species in $U$ and/or in $V$ ). 
1. For two identical plots $U$ and $V$, the ordinariness $z_{U j}$ of species $j$ in plot $U$ is equal to the ordinariness $z_{V j}$ of species $j$ in plot $V$.

For two identical plots, $x_{U j}=x_{V j}$ for all $N$ species in $U$ and $V$. Therefore, since $z_{U j}=\sum_{i=1}^{N} x_{U i} \sigma_{i j}$ (see Eq. 11 in the main text):

$$
z_{U j}=\sum_{i=1}^{N} x_{U i} \sigma_{i j}=\sum_{i=1}^{N} x_{V i} \sigma_{i j}=z_{V j}
$$

2. For two functionally identical plots $U$ and $V$ with no shared species, the ordinariness of species $j$ in plot $U$ is equal to the ordinariness of species $j$ in plot $V$.

Consider the simplest case where $U$ does not contain functionally identical species such that for all $i$ and $j$ in $U, \sigma_{i j}<1$, and similarly $V$ does not contain functionally identical species such that for all $m$ and $n$ in $V, \sigma_{m n}<1$. In that case, functionally identical plots necessarily have the same number of species. In addition, for each pair of species $i$ and $j$ in $U$, we have a functionally identical pair of species $m$ and $n$ in $V$ such that $x_{U i}=x_{V m}, \sigma_{i m}=1, x_{U j}=x_{V n}, \sigma_{j n}=1$ and for all $k$ in $\mathrm{U}$ and in $\mathrm{V}$, $\sigma_{i k}=\sigma_{m k}$ and $\sigma_{k j}=\sigma_{k n}$. Therefore, we have:

$$
z_{U j}=\sum_{i \in U} x_{U i} \sigma_{i j}
$$

and

$$
z_{V j}=z_{V n}=\sum_{m \in V} x_{V m} \sigma_{m n}=\sum_{i \in U} x_{U i} \sigma_{i n}=\sum_{i \in U} x_{U i} \sigma_{i j}=z_{U j}
$$

The demonstration for the more general case (where some species are allowed to be functionally identical within $U$ and/or within $V$ ) can simply be obtained by pooling a set of functionally identical species within a plot into a single super-species with its abundance equal to the summed abundance of the functionally identical species. Indeed, the ordinariness values of functionally identical species in a plot are equal to each other, and are also equal to the ordinariness of the super-species (see proof at point 4 below, Eq. A8 and A9). For example, let $J=\left\{j, j^{\prime}, j^{\prime \prime}, \ldots\right\}$ be such a super-species in $U$, such that $x_{U J}=x_{U j}+x_{U j^{\prime}}+x_{U j^{\prime \prime}}+\ldots$. If $U$ and $V$ are functionally identical, then there exist a species or a set of functionally identical species $K$ in $V$ such that $x_{U J}=x_{V K}$ and $\sigma_{J K}=1$.

3. For two maximally distinct plots with no species in common and with $\sigma_{j m}=0$ for species $j$ belonging to plot $U$ and species $m$ to plot $V, z_{V j}$ is always equal to zero, meaning that the species in $U$ do not have any functional analogue in $V$, and $z_{U m}$ is always equal to zero, meaning that the species in $V$ do not have any functional analogue in $U$

Observing that

$$
z_{V j}=\sum_{m \in V} x_{V m} \sigma_{j m}
$$


and

$$
z_{U m}=\sum_{j \in U} x_{U j} \sigma_{j m}
$$

460

461

462

463

464

465

Since $\sigma_{j m}=0$ by definition, we have $z_{V j}=z_{U m}=0$.

4. The general family of functional dissimilarity measures $D_{w}=\sum_{j=1}^{N} w_{j} d\left(z_{U j}, z_{V j}\right)$, with $w_{j}=\left(x_{U j}+x_{V j}\right) / \sum_{k=1}^{N}\left(x_{U k}+x_{V k}\right)$ (see Eq. 5 and 12 in the main text) conforms to the following branching requirement: the value of functional dissimilarity $D_{w}$ among a pair of plots $U$ and $V$ does not change if a given species $j$ is replaced by two functionally identical species with the same total abundance of $j$ in $U$ and $V$.

If species $j$ is split into two species $j^{\prime}$ and $j^{\prime \prime}$ which are functionally identical to $j$ such that $x_{U j^{\prime}}+x_{U j^{\prime \prime}}=x_{U j}, \quad x_{V j^{\prime}}+x_{V j^{\prime \prime}}=x_{V j}$. Because $j, \quad j^{\prime}$ and $j^{\prime \prime}$ are functionally identical $\sigma_{j^{\prime} j^{\prime \prime}}=\sigma_{j^{\prime} j}=\sigma_{j^{\prime \prime j}}=1$, and for any species $i$ in $U$ and/or $V, \sigma_{i j}=\sigma_{i j^{\prime}}=\sigma_{i j^{\prime \prime}}$. In addition, we have:

$w_{j^{\prime}}+w_{j^{\prime \prime}}=w_{j}$

and

$z_{U j}=\sum_{i}^{N} x_{U i} \sigma_{i j}=\sum_{i}^{N} x_{U i} \sigma_{i j^{\prime}}=\sum_{i}^{N} x_{U i} \sigma_{i j^{\prime \prime}}$

It follows that

$z_{U j^{\prime}}=z_{U j^{\prime \prime}}=z_{U j}$

and similarly

$z_{V j^{\prime}}=z_{V j^{\prime \prime}}=z_{V j}$

This yields

$d\left(z_{U j^{\prime}}, z_{V j^{\prime}}\right)=d\left(z_{U j^{\prime \prime}}, z_{V j^{\prime \prime}}\right)=d\left(z_{U j}, z_{V j}\right)$

Therefore, since $d\left(z_{U j^{\prime}}, z_{V j^{\prime}}\right) w_{j^{\prime}}+d\left(z_{U j^{\prime \prime}}, z_{V j^{\prime \prime}}\right) w_{j^{\prime \prime}}=d\left(z_{U j}, z_{V j}\right) w_{j}$, it follows that $D_{w}$ does not change if a given species $j$ is split into two functionally identical species.

Appendix 2. R scripts

The R function "generalized Tradidiss" calculates plot-to-plot dissimilarity taking account of functional dissimilarities between species. This program is free software: you can redistribute it 
and/or modify it under the terms of the GNU General Public License http://www.gnu.org/licenses/. It will be integrated in a version of the adiv package after the publication of the associated paper.

Disclaimer: users of this code are cautioned that, while due care has been taken and it is believed accurate, it has not been rigorously tested and its use and results are solely the responsibilities of the user.

Description: given a matrix of $S$ species' relative or absolute abundance values $\times N$ plots, together with an $S \times S$ (functional) dissimilarity matrix, the function "generalized_Tradidiss" calculates a semimatrix with the values of the dissimilarity index for each pair of plots, as proposed in the main text.

\section{Dependencies: none.}

\section{Usage:}

generalized_Tradidiss <-

function(comm, dis, method = c("GC", "MS", "PE"), abundance = c("relative", "absolute", "none"), weights = c("uneven", "even"), tol = 1e-8)

\section{Arguments:}

comm: a matrix of $N$ plots $\times S$ species containing the relative or absolute abundance of all species. Columns are species and plots are rows. Column labels (species names) should be assigned as in the dis matrix.

dis: an $S \times S$ matrix of dissimilarities between species rescaled in the range $[0,1]$ or an object of class "dist" [obtained by functions like vegdist in package vegan (Oksanen et al. 2013), gowdis in package FD (Laliberté \& Shipley 2011), or dist.ktab in package ade4 for functional dissimilarities (Dray et al. 2007), or functions like cophenetic.phylo in package ape (Paradis et al. 2004) or distTips in package adephylo (Jombart and Dray 2010) for phylogenetic dissimilarities]. If the dissimilarities are outside the range $0-1$, a warning message is displayed and each dissimilarity is divided by the maximum over all pair-wise dissimilarities.

method: a character string giving the name of the coefficient of plot-to-plot dissimilarity to be applied to species ordinariness (Z vectors defined in the main text, Eq. 11). It can be "GC", "MS", or "PE". See details.

abundance: a character string with three possible values: "relative" for the use of relative species abundance, "absolute" for the use of absolute species abundance, and "none" for the use of presence/absence data $(1 / 0)$.

weights: Two types of weights are available in the function: "uneven" (Eq. 5 in the main text) or "even" (1/S, where $\mathrm{S}$ is the number of species in the two compared plots)

tol: a numeric tolerance threshold: a value between -tol and tol is considered as null. 
Details:

The plot-to-plot dissimilarity coefficients used in this function are those listed in the main text. Codes are as follows:

\section{Value:}

The function returns a semi-matrix of class "dist" with the values of the proposed dissimilarities for each pair of plots.

\section{Function Syntax}

generalized_Tradidiss <-

function (comm, dis, method = c("GC", "MS", "PE"), abundance = c("relative", "absolute", "none"), weights =c("uneven", "even"), tol = $1 e-8)$ 


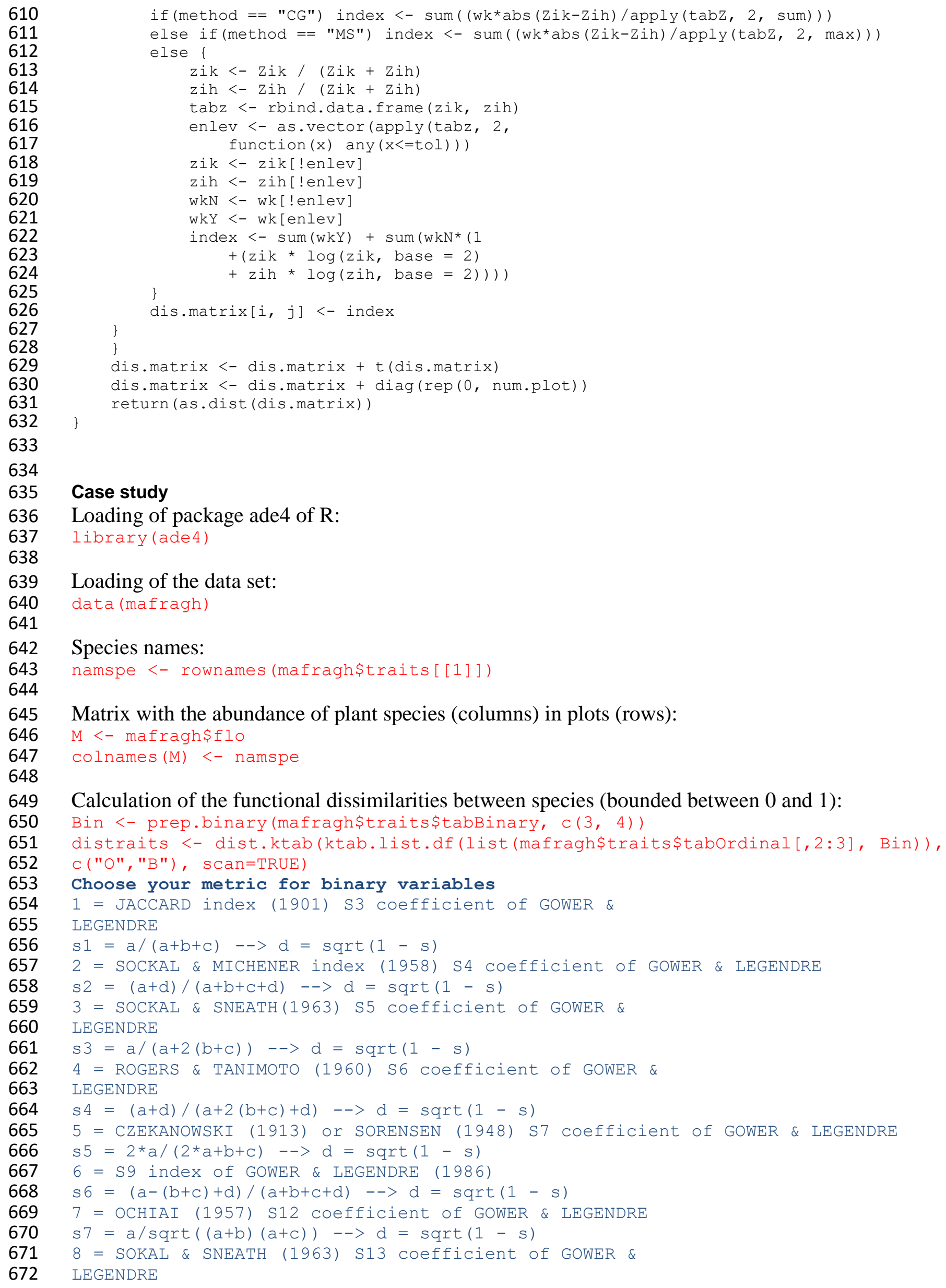

\section{Case study}

Loading of package ade 4 of R:

library (ade4)

Loading of the data set:

data (mafragh)

\section{Species names:}

namspe <- rownames (mafragh\$traits [1] ])

Matrix with the abundance of plant species (columns) in plots (rows):

$\mathrm{M}<-$ mafragh\$flo

colnames $(\mathrm{M})<-$ namspe

Calculation of the functional dissimilarities between species (bounded between 0 and 1):

Bin <- prep.binary (mafragh\$traits\$tabBinary, c (3, 4))

distraits <- dist.ktab(ktab.list.df(list(mafragh\$traits\$tabordinal[,2:3], Bin)), c ("O","B"), scan=TRUE)

Choose your metric for binary variables

1 = JACCARD index (1901) S3 coefficient of GOWER \&

LEGENDRE

$\mathrm{s} 1=\mathrm{a} /(\mathrm{a}+\mathrm{b}+\mathrm{c})-->\mathrm{d}=\operatorname{sqrt}(1-\mathrm{s})$

2 = SOCKAL \& MICHENER index (1958) S4 coefficient of GOWER \& LEGENDRE

$\mathrm{s} 2=(\mathrm{a}+\mathrm{d}) /(\mathrm{a}+\mathrm{b}+\mathrm{c}+\mathrm{d})-->\mathrm{d}=\operatorname{sqrt}(1-\mathrm{s})$

3 = SOCKAL \& SNEATH(1963) S5 coefficient of GOWER \&

LEGENDRE

$\mathrm{s} 3=\mathrm{a} /(\mathrm{a}+2(\mathrm{~b}+\mathrm{c}))-->\mathrm{d}=\operatorname{sqrt}(1-\mathrm{s})$

$4=$ ROGERS \& TANIMOTO (1960) S6 coefficient of GOWER \&

LEGENDRE

$\mathrm{s} 4=(\mathrm{a}+\mathrm{d}) /(\mathrm{a}+2(\mathrm{~b}+\mathrm{c})+\mathrm{d})-->\mathrm{d}=\operatorname{sqrt}(1-\mathrm{s})$

5 = CZEKANOWSKI (1913) or SORENSEN (1948) S7 coefficient of GOWER \& LEGENDRE

$\mathrm{s} 5=2 * a /(2 * a+b+c)-->d=\operatorname{sqrt}(1-\mathrm{s})$

$6=\mathrm{S} 9$ index of GOWER \& LEGENDRE (1986)

$\mathrm{s} 6=(\mathrm{a}-(\mathrm{b}+\mathrm{c})+\mathrm{d}) /(\mathrm{a}+\mathrm{b}+\mathrm{c}+\mathrm{d})-->\mathrm{d}=\operatorname{sqrt}(1-\mathrm{s})$

7 = OCHIAI (1957) S12 coefficient of GOWER \& LEGENDRE

$\mathrm{s} 7=\mathrm{a} / \operatorname{sqrt}((\mathrm{a}+\mathrm{b})(\mathrm{a}+\mathrm{c}))-->\mathrm{d}=\operatorname{sqrt}(1-\mathrm{s})$

8 = SOKAL \& SNEATH (1963) S13 coefficient of GOWER \& LEGENDRE 


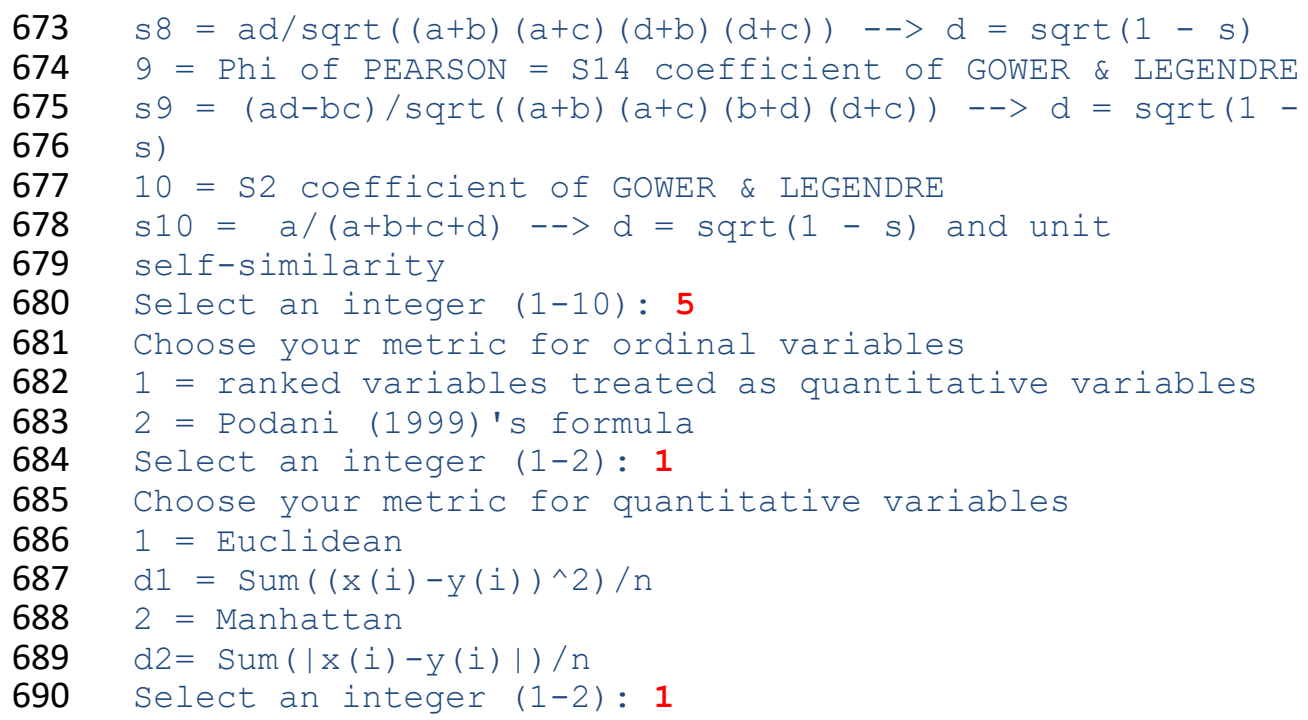

Application of the Bray-Curtis index (Eq. 4 of the main text) or equivalently index GC (Eq. 6) with uneven weights for species (Eq. 5) to the data set:

disGC <- generalized_Tradidiss(M, distraits, method="GC")

The functional dissimilarities between plots do not have the Euclidean properties is.euclid (as.dist (disGC))

[1] FALSE

First approach: Principal coordinate analysis (PCoA) on the functional dissimilarities transformed by Cailliez approach (addition to the dissimilarities of the smallest constant that renders the dissimilarities Euclidean)

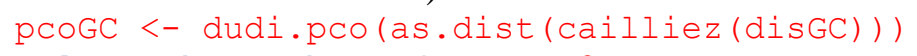

Coordinates of the species on the first axis of the PCoA (given by the color and size of a square) at their spatial location in the plain La Mafragh (a square is at the geographic position of its associated plot):

s.value (mafragh\$xy, pcoGC\$1i $[, 1]$ ) 


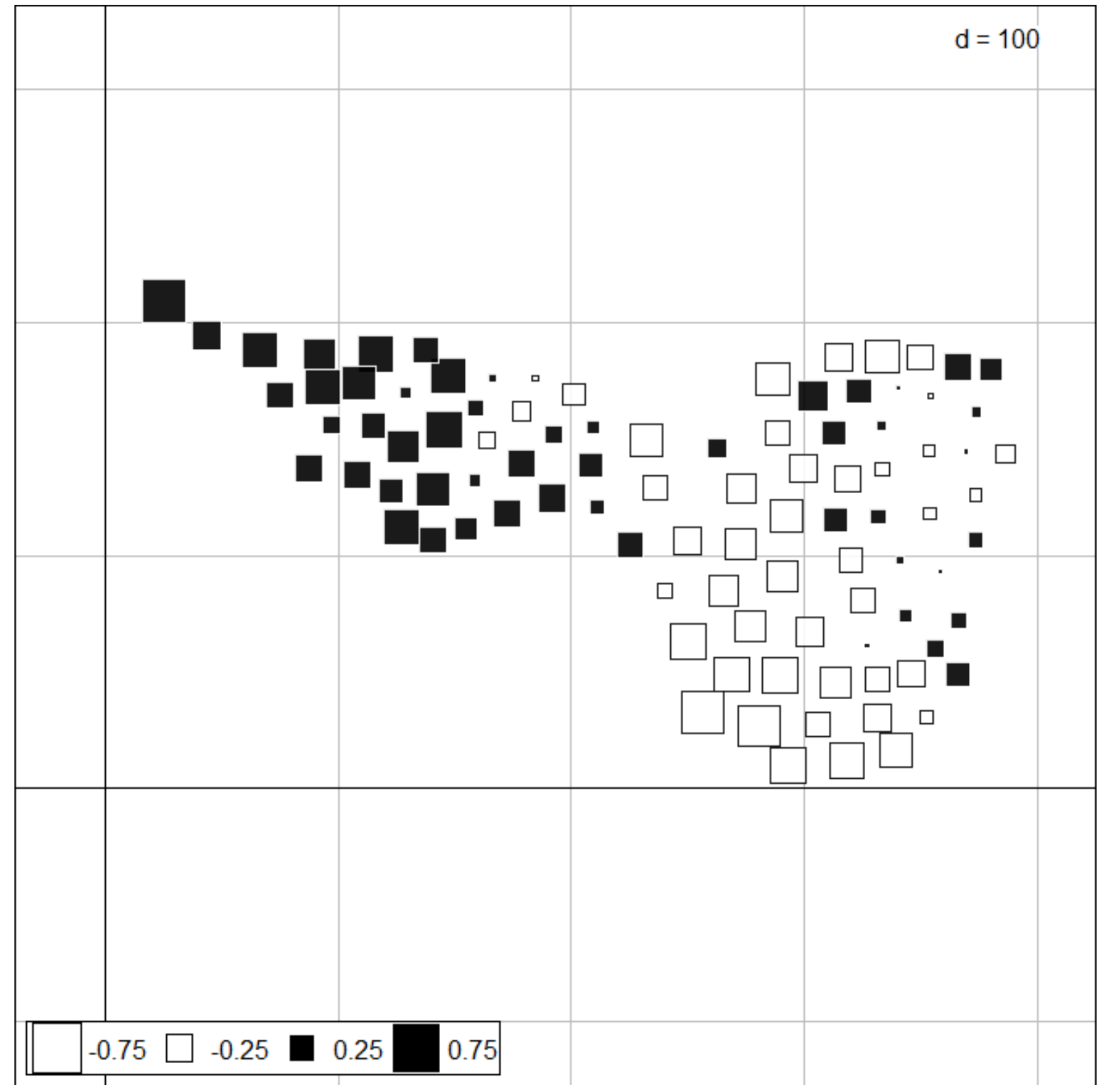

Second approach: non-metric Multidimensional Scaling analysis (nMDS) on the functional dissimilarities

CMD $<-$ cmdscale (disGC)

summary (CMD)

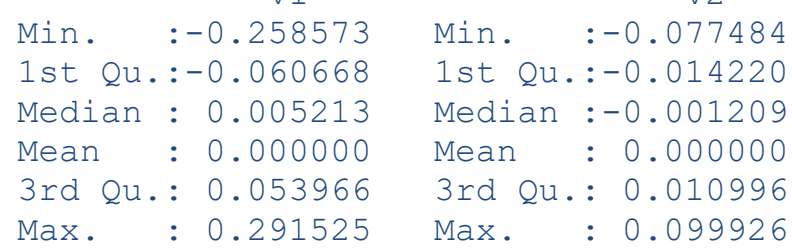

The coordinates on the second axis of the nMDS are low indicating that most of the functional variation between plots is expressed by the first axis.

Spatial pattern of the first axis of the nMDS: 


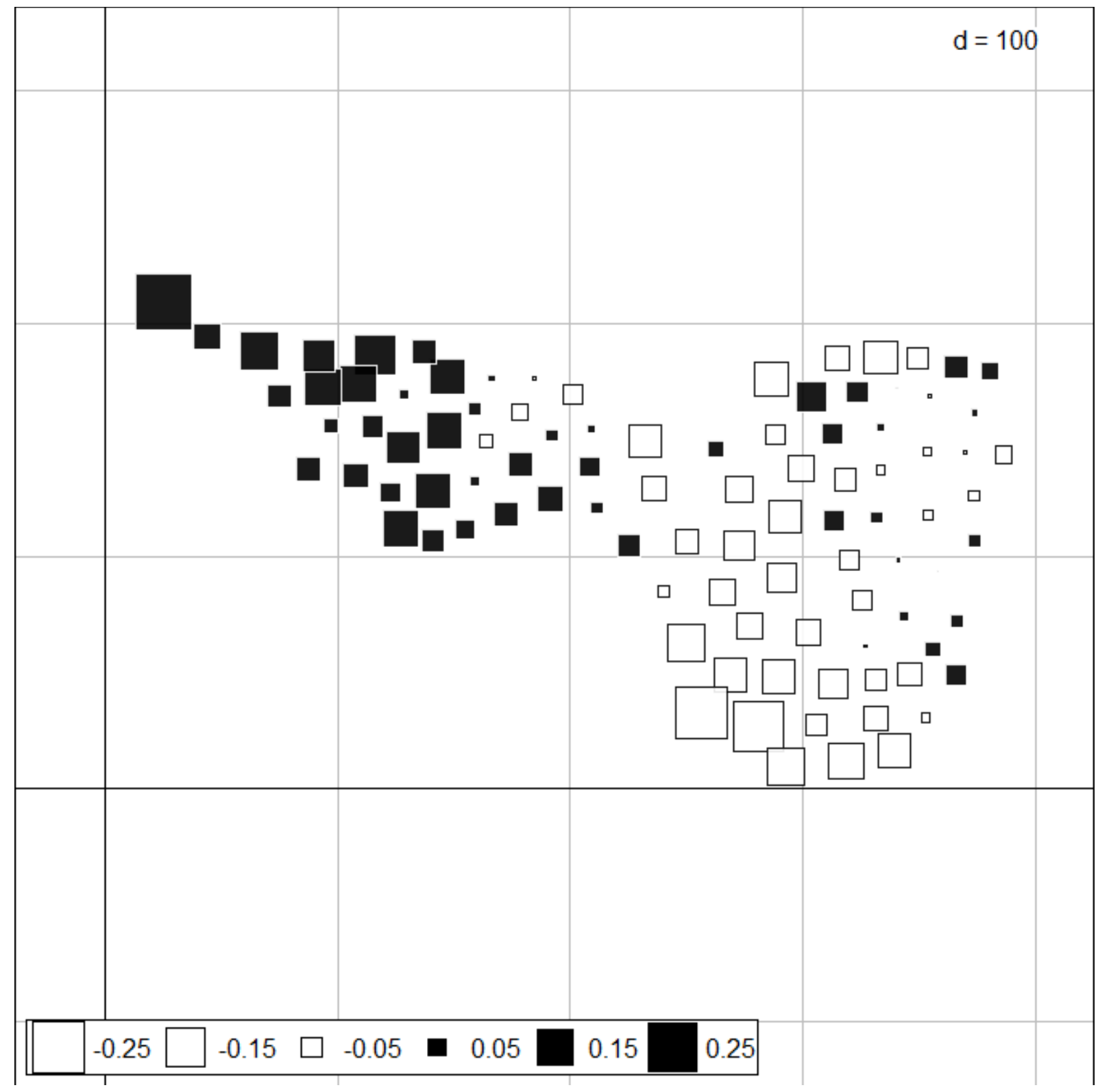

Link between the coordinates of plots in PCoA and those in the nMDS: 


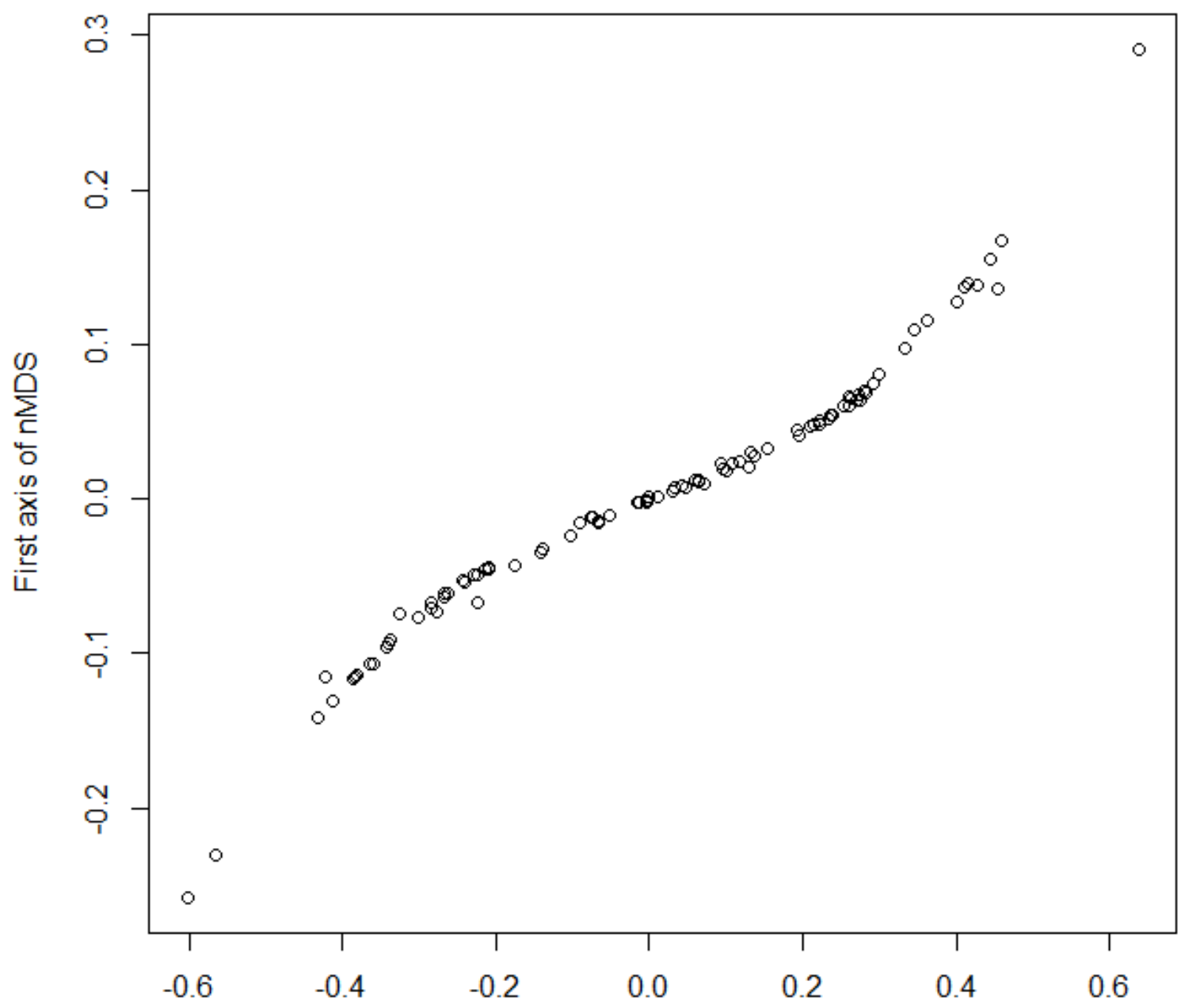

First axis of $\mathrm{PCoA}$

\section{Analysis of the species-based dissimilarities between plots:}

Species are defined as maximally dissimilar:

DM1 <- as.dist (matrix $(1,56,56)$ )

attributes (DM1) \$Labels <- attributes (distraits) \$Labels

Application of the Bray-Curtis index (Eq. 4 of the main text) or equivalently index GC (Eq. 6) with uneven weights for species (Eq. 5) to the data set with species defined as maximally dissimilar:

disM1 <- generalized_Tradidiss (M, DM1)

The species-based dissimilarities between plots do not have the Euclidean properties

is.euclid (disM1)

[1] FALSE

Principal Coordinate Analysis of the species-based dissimilarities between plots pcoDM1 <- dudi.pco(as.dist(cailliez(disM1)))

Select the number of axes: 2 


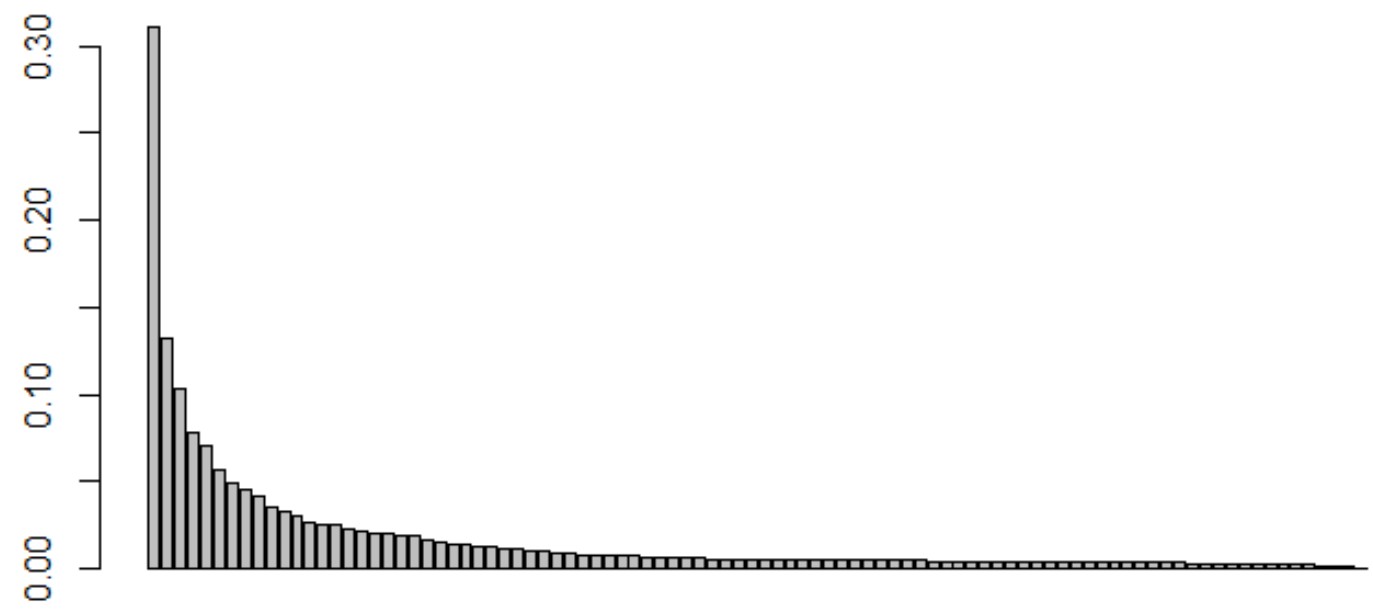

Coordinates of the species on the first axis of the PCoA (given by the color and size of a square) at their spatial location in the plain La Mafragh (a square is at the geographic position of its associated plot):

770 s.value (mafragh\$xy, pcoDM1\$li[,1], csize=0.5) 


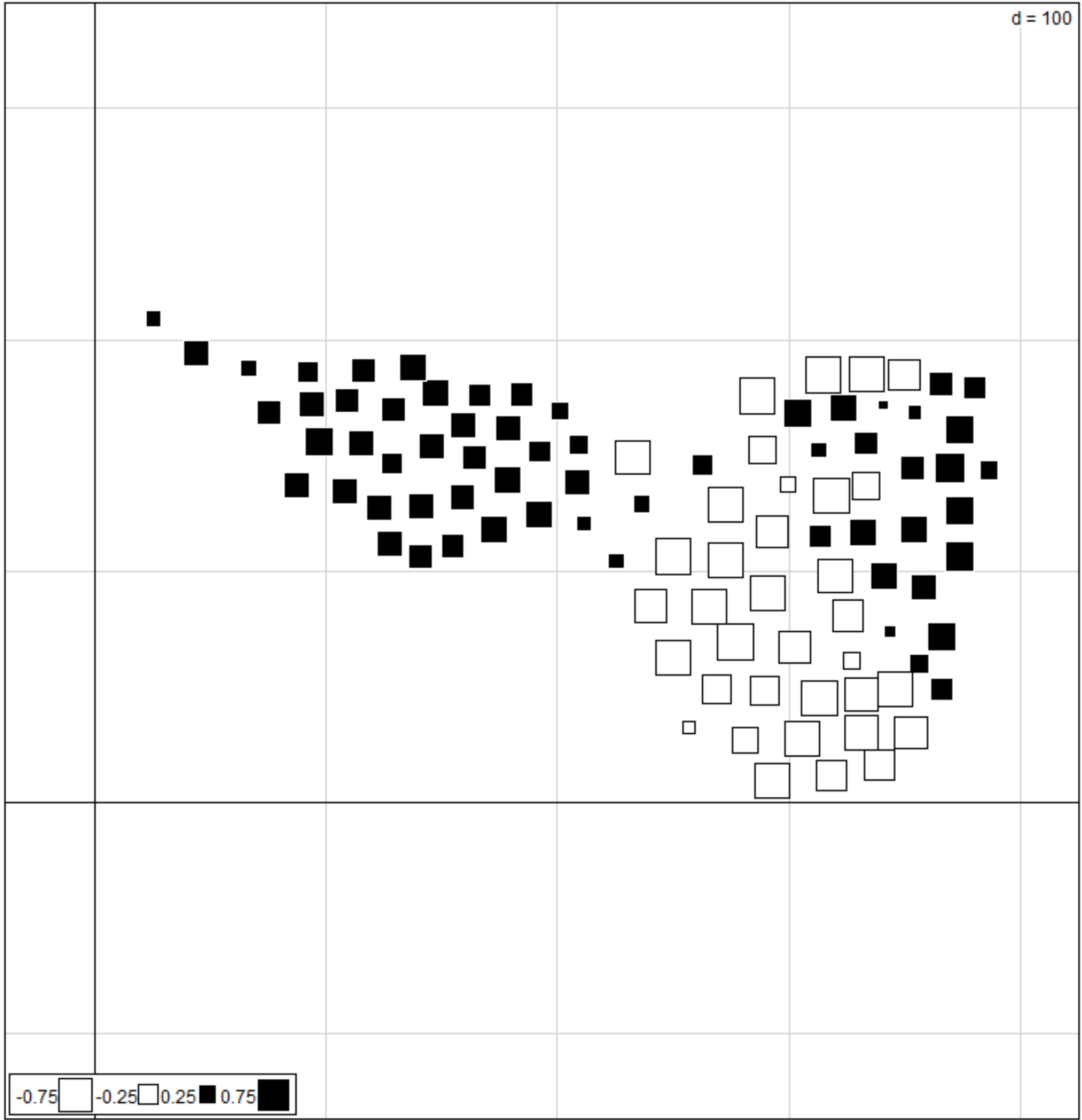

779 


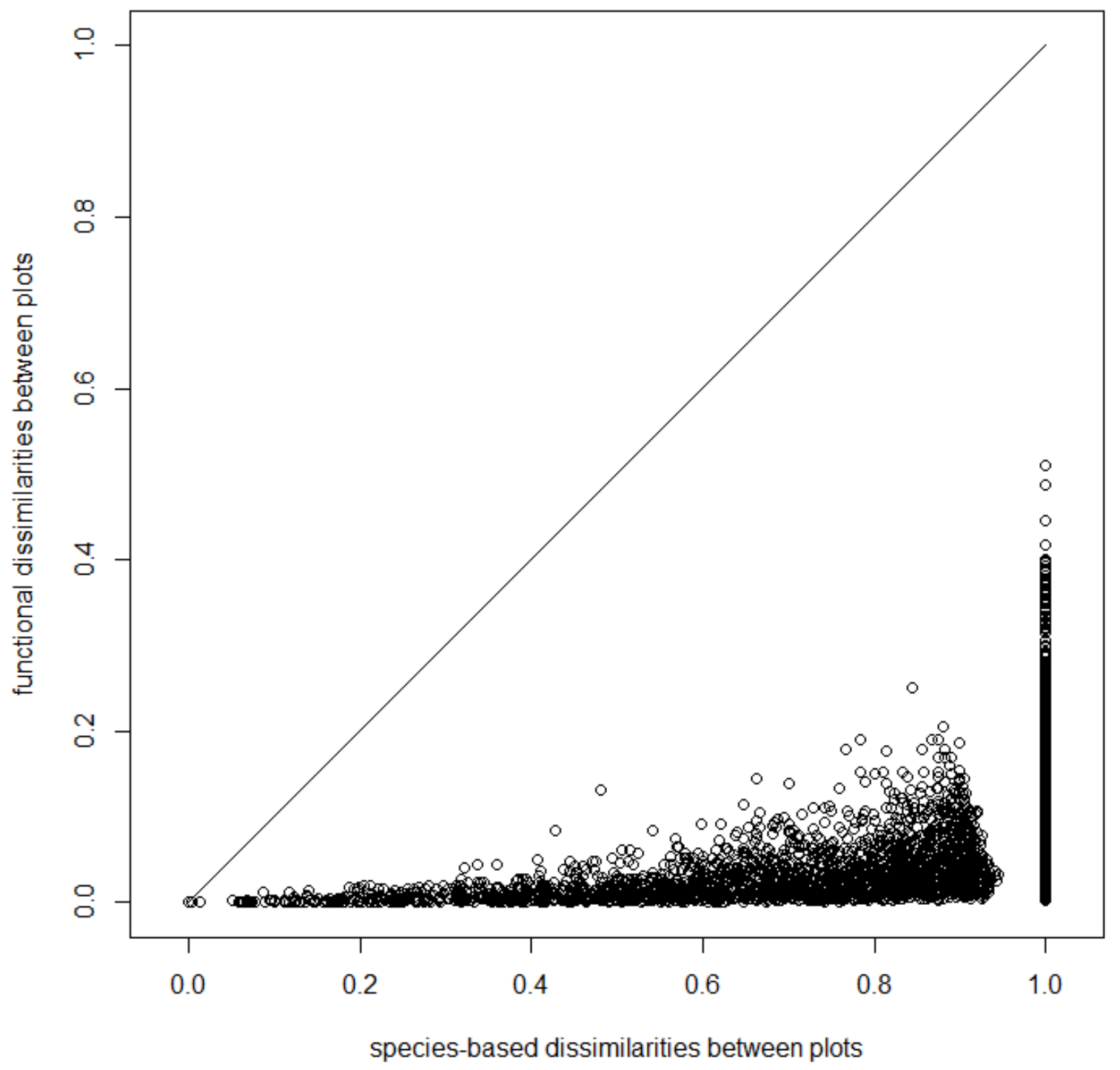

Analysis of environmental data on the factorial map of the functional PCoA:

Environmental data:

env $<-$ mafragh $\$$ env $[-(8: 10)]$

Correlations between environmental data and the coordinates of plots on the first axis of the functional PCoA:

cor(env, pcoGC\$li[,1], method="spearman")

Clay $\quad-0.3441044$

Silt $\quad 0.2214038$

Sand $\quad 0.4734112$

K2O $\quad-0.3989277$

$\mathrm{Mg}++\quad-0.2372428$

$\mathrm{Na}+100 \mathrm{~g} \quad-0.5128574$

K+ $\quad-0.6414609$

Elevation 0.3561348 


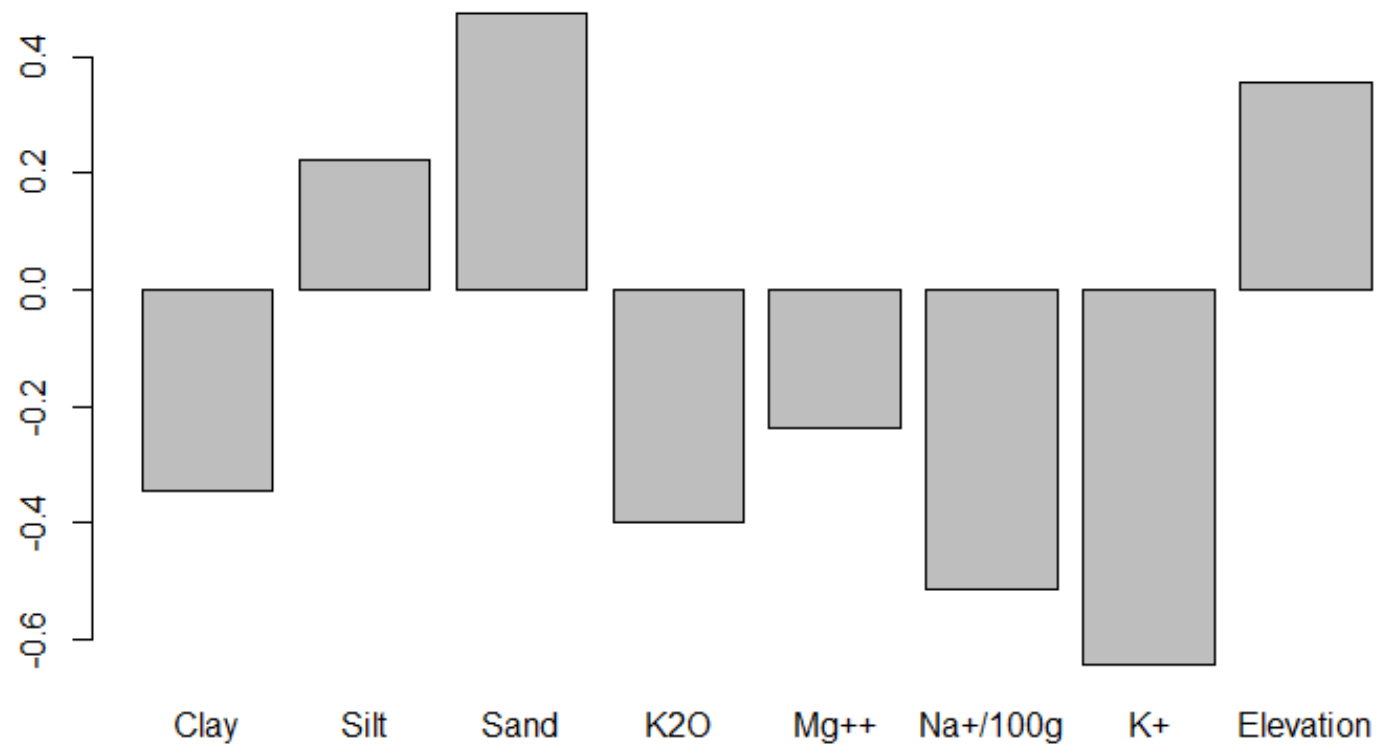

\section{References}

Dray, S., Dufour, A.B., Chessel, D. (2007) The ade4 package-II: Two-table and K-table methods. R News 7: 47-52.

Jombart, T., Dray S. (2008) adephylo: exploratory analyses for the phylogenetic comparative method. Bioinformatics 26: 1907-1909.

Laliberté, E., Legendre, P. (2010) A distance-based framework for measuring functional diversity from multiple traits. Ecology 91: 299-305.

Oksanen, J., Blanchet, F.G., Friendly, M., Kindt, R., Legendre, P., McGlinn, D., Minchin P.R., O'Hara, R.B., Simpson, G.L., Solymos, P., Henry, M., Stevens, H., Szoecs, E., Wagner, H. (2018) vegan: Community Ecology Package. R package version 2.4-6. https://CRAN.Rproject.org/package=vegan.

Paradis, E., Claude, J., Strimmer, K. (2004) APE: analyses of phylogenetics and evolution in R language. Bioinformatics 20: 289-290.

Appendix 3. On the relationship between species ordinariness and species rarity

Let $U$ and $V$ be two plots (or communities, assemblages, relevés, sites, quadrats, etc.) where $x_{U j}$ and $x_{V j}$ are the abundance values of species $j$ in plots $U$ and $V$, respectively, $p_{U j}=x_{U j} / \sum_{j=1}^{N} x_{U j}$ is the relative abundance of species $j$ in plot $U$ (with $0 \leq p_{U j} \leq 1$ and $\sum_{j=1}^{N} p_{U j}=1$ ), and $N$ is the total number of species sampled in both plots (i.e. the species for which $\min \left\{x_{U j}, x_{V j}\right\}>0$ ). Leinster and Cobbold (2012) defined the ordinariness of species $j$ in plot $U$ as: 
where $z_{U j}$ is the abundance of all species that are functionally similar to $j$ (including $j$ itself), and $\sigma_{i j}$ is a symmetric similarity coefficient between species $i$ and $j$ such that $\sigma_{i j}=\sigma_{j i}$ and $\sigma_{j j}=0$. For species $j, z_{U j}$ thus measures the commonness of all individuals in plot $U$ that support the functions associated with $j$.

In the main text, we showed that the quantity $z_{U j}$ can be used for deriving a general family of functional dissimilarity coefficients among plots $U$ and $V$ (see Eq. 10 of the main text). Here, we show that if species ordinariness $z_{U j}$ is calculated from the species relative abundances $p_{U j}$ such that

$z_{U j}=\sum_{i=1}^{N} p_{U i} \sigma_{i j}$

the resulting measure is tightly related to the notion of species rarity used by Patil and Taillie (1982) for providing a general definition of diversity coefficients.

Rao (1982) introduced a diversity index, termed Quadratic diversity, for summarizing mean interspecies differences within a given plot. The quadratic diversity of plot $U$ is defined as the expected dissimilarity between two individuals drawn randomly with replacement from the plot:

$Q_{U}=\sum_{j=1}^{N} \sum_{i=1}^{N} p_{U i} p_{U j} \delta_{i j}$

where $\delta_{i j}$ is the dissimilarity among species $i$ and $j$.

For a review of some relevant mathematical properties of Rao's $Q$ see Pavoine (2012). Here, it is sufficient to observe that if $\delta_{i j}$ is bounded between 0 and 1, assuming that all species are maximally distinct from one another (i.e. if $\delta_{i j}=1$ for all $i \neq j$ ), $Q_{U}$ reduces to the classical Simpson index of diversity $S_{U}=1-\sum_{j=1}^{N} p_{U j}^{2}$. Also, because $0 \leq \delta_{i j} \leq 1$, an associated measure of concentration or dominance (i.e. the opposite of diversity) can be defined as the complement of Rao's Q (Leinster and Cobbold 2012):

$D_{U}=1-Q_{U}=\sum_{j=1}^{N} \sum_{i=1}^{N} p_{U i} p_{U j} \sigma_{i j}$

where $D_{U}$ is the average similarity between all species in plot $U$, and $\sigma_{i j}=1-\delta_{i j}$.

To gain insight into the type of diversity measured by the Simpson and the Rao coefficients, it is convenient to express Simpson in the form $S_{U}=\sum_{j=1}^{N} p_{U j}\left(1-p_{U j}\right)$. In the context of interspecific encounter theory, Patil and Taillie (1982) defined the Simpson diversity as the probability that two individuals drawn randomly from a given plot do not belong to the same species. Therefore, the Simpson diversity is essentially a measure of uncertainty in predicting the relative abundance of the species in the plot. In information theory and statistics, the quantity $\left(1-p_{U j}\right)$ is usually referred to as the surprise or rarity associated to species $j$ (Ricotta and Szeidl 2006; Leinster and Cobbold 2012). If $p_{U j}$ is small, we would be quite surprised in finding species $j$. To the contrary, if $p_{U j}$ is 
large, the surprise in finding $j$ is small. Patil and Taillie (1982) thus proposed a general definition of diversity $(\Delta)$ as the average rarity $\rho\left(p_{U j}\right)$ of a given assemblage

$$
\Delta=\sum_{j=1}^{N} p_{U j} \rho\left(p_{U j}\right)
$$

where $\rho\left(p_{U j}\right)$ is some decreasing function of the species relative abundance $p_{U j}$.

Klir and Wierman (1999) and Ricotta and Szeidl (2006) noted that the quantity $\left(1-p_{U j}\right)=\sum_{i \neq j} p_{U i}$ measures the relative abundance of all species that differ from species $j$. That is, the probabilities of all events that conflict with $p_{U j}$. Accordingly, assuming that all species are maximally distinct from each other (i.e. if $\delta_{i j}=1$ for all $i \neq j$ ), the Simpson index can be interpreted as a measure of the expected conflict $C\left(p_{U j}\right)$ of all species in the assemblage (Ricotta and Szeidl 2006):

$$
S_{U}=\sum_{j=1}^{N} p_{U j} \sum_{i \neq j}^{N} p_{U i}=\sum_{j=1}^{N} p_{U j} C\left(p_{U j}\right)
$$

Relaxing the constraint of maximal dissimilarity among species, it seems natural to summarize the conflict of species $j$ with the other species in the assemblage taking into account their pairwise distances $\delta_{i j}$ such that:

$$
C\left(p_{U j}\right)=\sum_{j=1}^{N} p_{U j} \delta_{i j}
$$

with $\delta_{j j}=0$. Accordingly, incorporating a measure of pairwise dissimilarity among species, the Simpson expression of expected conflict becomes Rao's quadratic diversity $\sum_{j=1}^{N} p_{U j} \sum_{i=1}^{N} p_{U i} \delta_{i j}$. Rao's $Q$ can be thus interpreted as a general measure of expected rarity or conflict in a given assemblage if the species are not treated as maximally distinct from each other (Ricotta and Szeidl 2006).

Likewise, we can define the ordinariness of species $j$ as the complement of its conflict:

$$
z_{U j}=1-C\left(p_{U j}\right)=\sum_{j=1}^{N} p_{U j} \sigma_{i j}
$$

such that the expected species concentration in Eq. A4 can be rewritten as $D_{U}=\sum_{j=1}^{N} p_{U j} \sum_{i=1}^{N} p_{U i} \sigma_{i j}=\sum_{j=1}^{N} p_{U j} z_{U j}$, while Rao's diversity becomes $Q_{U}=1-\sum_{j=1}^{N} p_{U j} z_{U j}$, where the ordinariness $z_{U j}$ is the expected similarity between an individual of species $j$ and an individual drawn randomly from the assemblage.

Therefore, according to the above derivation, if the values of $z_{U j}$ are calculated from the species relative abundances $p_{U j}$, species ordinariness may represent a common currency for expressing both the species functional diversity in single plots and their functional dissimilarity among pair of plots.

\section{References}


Klir, G.J., Wierman, M.J. (1999) Uncertainty-Based Information. Physica-Verlag, Heidelberg.

Leinster, T., Cobbold, C.A. (2012) Measuring diversity: the importance of species similarity. Ecology 93: 477-489.

Patil, G.P., Taillie, C. (1982) Diversity as a concept and its measurement. Journal of the American Statistical Association 77: 548-561.

Pavoine, S. (2012) Clarifying and developing analyses of biodiversity: towards a generalisation of current approaches. Methods in Ecology and Evolution 3: 509-518.

Rao, C.R. (1982) Diversity and dissimilarity coefficients: a unified approach. Theoretical Population Biology 21: 24-43.

Ricotta, C., Szeidl, L. (2006) Towards a unifying approach to diversity measures: bridging the gap between the Shannon entropy and Rao's quadratic index. Theoretical Population Biology 70: 237-243. 